\title{
Calorimeter-less gamma-ray telescopes: Optimal measurement of charged particle momentum from multiple scattering by Bayesian analysis of Kalman filtering innovations.
}

\author{
Denis Bernard \\ LLR, Ecole polytechnique, 91128 Palaiseau, France \\ E-mail: denis.bernardein2p3.fr

\section{Mikael Frosini} \\ LLR, Ecole polytechnique, 91128 Palaiseau, France
}

\begin{abstract}
Novel gamma-ray telescope schemes (silicon wafer stacks, emulsions, gas detectors) are being developed so as to bridge the sensitivity gap between Compton and pair-creation telescopes. The lower average density with respect to the tungsten/silicon active target of the Fermi-LAT makes large effective-area telescopes voluminous objects, for which the photon energy measurement by conventional means (calorimeter, magnetic spectrometer, transition radiation detector) is a challenge for the mass budget of the space mission. We present an optimal measurement of track momentum by the multiple measurement of the angular deflections induced by multiple scattering in the active target itself, using a Bayesian analysis of the filtering innovations of a series of Kalman filters applied to the track. For a silicon-wafer-stack telescope, the method yields meaningful results up to a couple of $\mathrm{GeV} / \mathrm{c}$.
\end{abstract}

7th Fermi Symposium 2017

15-20 October 2017

Garmisch-Partenkirchen, Germany 
This work originated in the context of a theoretical and experimental development of low density, homogeneous detectors such as a gas time-projection chamber as a high-performance gammaray telescope and polarimeter in the $\gamma \rightarrow e^{+} e^{-}$regime, that is, above a photon energy of $1 \mathrm{MeV}$ [1]. In these "active targets", the incident $\gamma$ converts and the produced electrons are tracked in the same structure. The single-track angular resolution of these detectors, if tracking is performed with an optimal method in the presence of multiple scattering such as a Kalman filter [2], is so good that polarimetry has been predicted to be possible despite the dilution of the polarization asymmetry induced by multiple scattering [3], and has actually been demonstrated by the characterization of a time-projection chamber (TPC) prototype on beam [4, 5]. Other projects plan to use all-silicon active targets, that is, without any additional tungsten converters [6, 7]. A third possibility is the use of emulsion active targets [8]. Whatever the method used, the lower density and the lower average atomic number of the active target, with respect to the tracker of the Fermi-LAT, imply a larger volume at a given value of the effective area: measuring the photon energy, be it with a calorimeter or by a measurement of the electron momenta with a magnetic spectrometer or with a transition radiation detector, is a challenge to the mission mass budget.

Lower energies are the domain where Compton telescopes are most efficient. When one scatter is detected, from the measurement of the position and the energy of the recoil electron and of the absorbed scattered photon, the reconstruction of the direction of the incident photon constrains it to a large arc, that is a large point-spread function (psf). If in addition the direction of the recoil electron is measured, the extension of the arc is reduced and the psf is improved [9]. Alas, the angular coverage of the calorimeter is an issue: it must be low enough that the incident photon can enter the detector and large enough to measure the energy of the scattered photon: in this electrontracking Compton camera (ETCC) scheme the effective area undergoes a sharp drop for photon energies above $0.5 \mathrm{MeV}[9]$.

The measurement of the angle deflections induced by multiple scattering of the electrons in the tracker has been used to yield an estimate of the track momentum since the 1950's [10]. Multiple scattering expresses the combined angle deflection induced by the multiple single deflections of a charged particle due to its many close encounters with the ions and with the electrons of matter as a Gaussian distribution with RMS

$$
\theta_{0} \approx \frac{p_{0}}{\beta p} \sqrt{\frac{\Delta x}{X_{0}}}\left(1+\varepsilon \log \frac{\Delta x}{X_{0}}\right)
$$

where $p_{0}=13.6 \mathrm{MeV} / c$ is the multiple-scattering constant, $\Delta x$ is the matter thickness through which the particle propagates and $X_{0}$ is its radiation length [11]. We neglect the small logarithmic correction term parametrized by $\varepsilon$.

As $\theta_{0}$ is inversely proportional to the track momentum $p$ and as each deflection measurement $\theta$ can be considered as an estimate of the RMS $\theta_{0}$, an estimate of the momentum can be obtained from multiple measurements of $\theta$ along the track [10]. Tracks are usually segmented into tracklets, each of which is fit so as to measure its angle. The comparison of the angles of successive tracklets yields the measurement of the deflection. The choice of the detector segmentation is of utmost importance in particular in the case of detectors that are physically segmented such as for silicon detectors. Shorter segments enable more deflection measurements but each of which with a larger angle uncertainty. A back-of-the-envelope guestimate of the optimal value of the tracklet length 
$\Delta$ was obtained [2], assuming that the expression of the relative momentum uncertainty $\sigma_{p} / p$ is the sum of the low- $\Delta$ and of the high- $\Delta$ asymptotes. It was found that the optimal segmentation depends on the value of the track momentum that we aim to measure.

Kalman-filter-based tracking is known to take into account the single-point spatial precision of the tracker and multiple scattering in an optimal way, but only at the condition that the "noise" covariance matrices, that in particular of the momentum-dependent multiple-scattering "process" noise, be known [12]. Combining an estimate $x_{k \mid k-1}$ of the track parameters at point $k$ obtained from the $k$ measurements $z_{i}, i=0 \cdots k-1$ and the measurement at point $k, z_{k}$, in an optimal way, given their covariance matrices, a Kalman filter obtains an optimal estimate of the track parameters, $x_{k \mid k}$, at point $k$; by recurrence an optimal estimate of the track parameters at the end of the track are obtained. The residues, $v_{k} \equiv z_{k}-x_{k \mid k-1}$ are named filtering innovations. For the optimal value of the Kalman filter gain, that is, for the correct value of the track momentum, the $v_{k}$ can be shown to be white, that is, uncorrelated, random variables.

How to devise a segmentation-free, optimal, unbiased, momentum measurement from the amazing ability of Kalman filters to (statistically) decipher the (detector precision) uncorrelated and the (multiple scattering) correlated interleaved contributions in the track trajectory statistics? We develop an optimal method to measure the electron momentum from the multiple measurement of multiple scattering induced deflections, based on a Bayesian analysis of the innovation residues of a set of momentum-dependent Kalman filters applied to the track [13]. ${ }^{1}$ Let's define $s$, the track-momentum-dependent multiple-scattering-angle average variance per unit track length:

$$
s \equiv\left(\frac{p_{0}}{p}\right)^{2} \frac{\Delta x}{l X_{0}} .
$$

It can be shown by the recurrence application of the Bayes theorem [14] that the probability $p_{n}(s)=p_{n}\left(z_{0} \cdots z_{n} \mid s\right)$ of observing the $n+1$ first measurements along the track, $z_{0} \cdots z_{n}$, given $s$, can be expressed as the product of the probability density functions of the innovations of a Kalmanfilter applied to $z_{0} \cdots z_{n} . l$ is the longitudinal sampling (in the case of a homogeneous detector, the thickness of the scatterer is equal to the length of the longitudinal sampling, $l=\Delta x$ ).

The distribution of the probability density function $p(s)$ is shown in Fig. 1 for one simulated $50 \mathrm{MeV} / c$ track [13]. The most probable value of $p$ is then obtained from the most probable value of $s: p=p_{0} \sqrt{\Delta x / l X_{0} s}$.

The method provides an optimal measurement of the process-noise parameters [14]. A numerical characterisation of the method shows that for a given detector the method is reliable up to some limit momentum above which the relative precision $\sigma_{p} / p$ becomes larger than unity. For lower momentum tracks, the momentum estimation is found to be unbiased (Fig. 2). The method

\footnotetext{
${ }^{1}$ This work has been performed under a number of assumptions/approximations. We assume relativistic particles ( $\beta \approx 1$ ) without loss of generality. Only the first-order term (angle deflection) of multiple scattering is taken into account which is legitimate for the thin detectors considered here; the 2nd-order transverse displacement is neglected. Continuous $(\mathrm{d} E / \mathrm{d} x)$ and discrete (Bremsstrahlung radiation) energy losses are also neglected. In TPCs in which the signal is sampled, most often the electronics applies a shaping of the pulse before digitisation, that creates a shortscale longitudinal correlation between successive measurements that we neglect too. Also the limitations of pattern recognition, that is, in the case of pair $\gamma$-ray telescopes, of the assignment of each hit to one of two close tracks, are not addressed.
} 

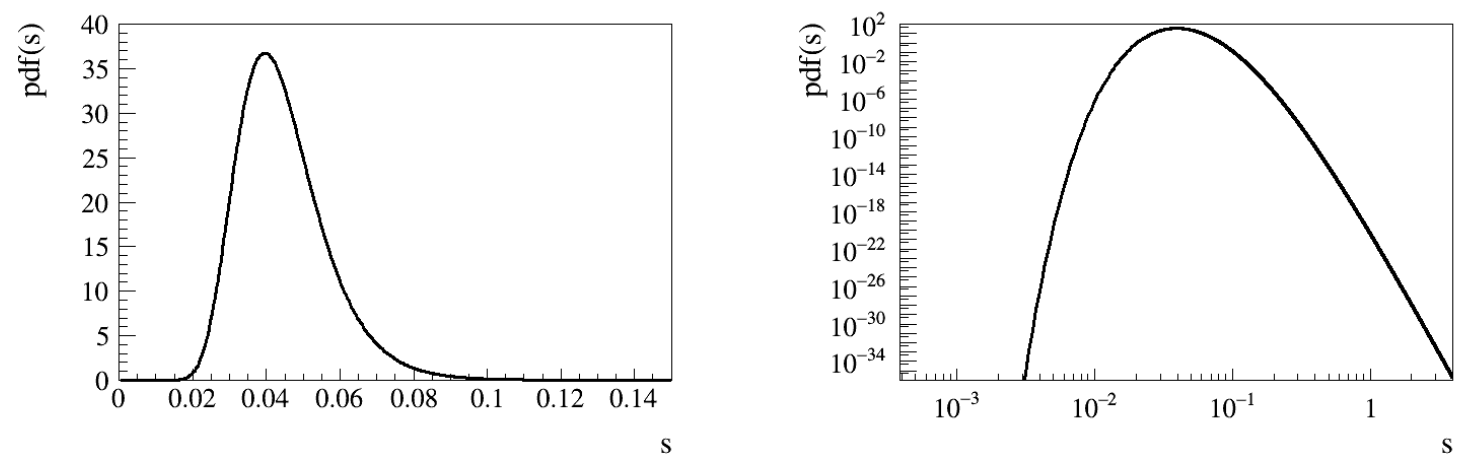

Figure 1: $p(s)$ distribution for a $50 \mathrm{MeV} / c$ track in a silicon detector. On that track, the momentum is measured to be equal to $49.9 \mathrm{MeV} / c$ [13] (left: lin-lin; right: $\log -\log$ ).

is found to be usable at low momentum, below a couple of $\mathrm{GeV} / c$ for silicon detectors such as e-ASTROGAM [6] or AMEGO [7].
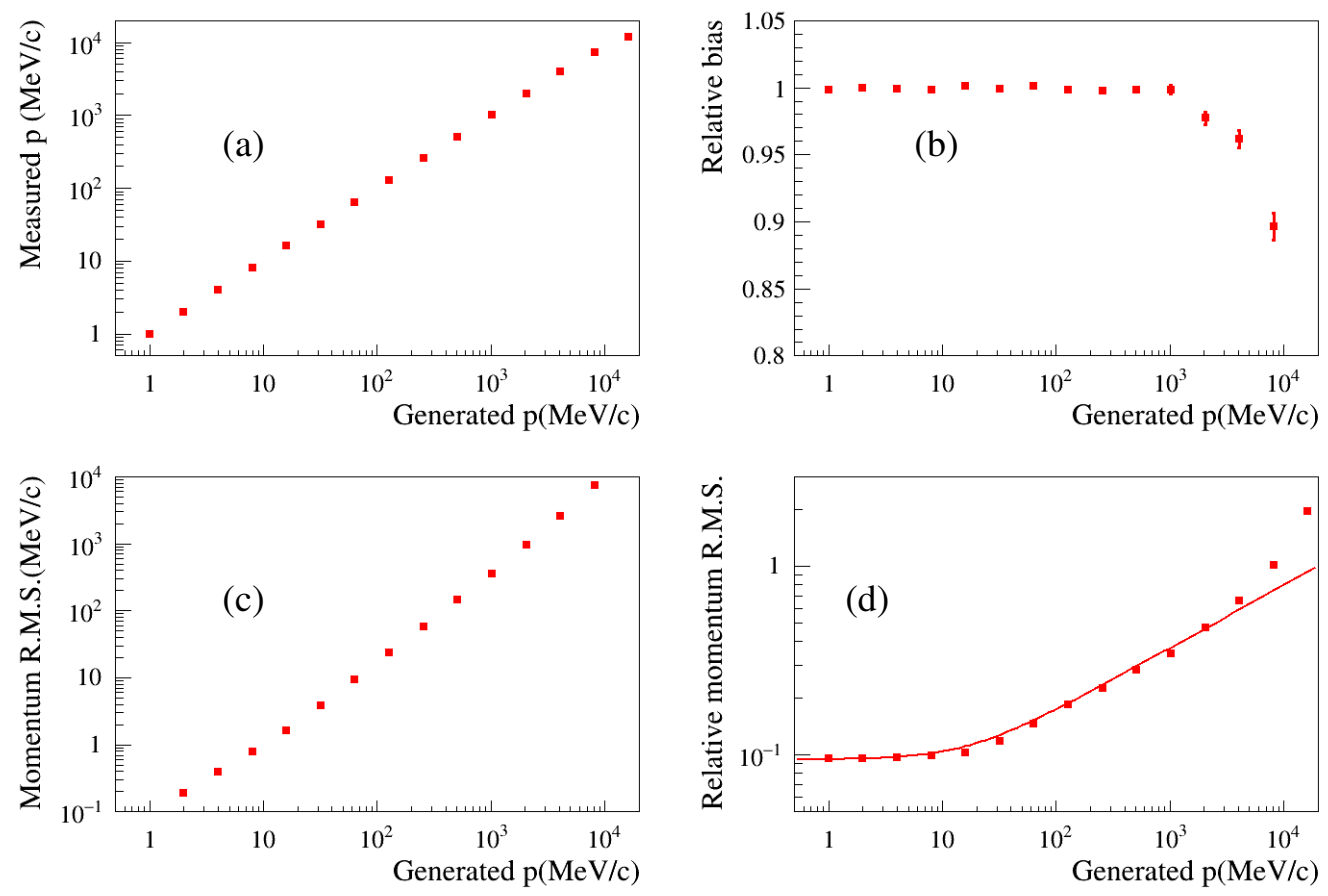

Figure 2: Performance of the momentum measurement for the silicon detector: Variation as a function of the true (generated) particle momentum of (a) the average measured momentum; (b) the average measured normalized to the generated momentum; (c) R.M.S of the measured momenta; (d) the relative R.M.S of the measured momenta [13]. The curve is from eq. (3). For each momentum value, the calculation is based on a sample of $10^{4}$ simulated tracks.

We perform a parametric study of the estimator from which we extract a heuristic analytical description of the relative uncertainty of the momentum measurement. A good representation of 
these data is obtained with the following expression [13]:

$$
\frac{\sigma_{p}}{p} \approx \frac{1}{\sqrt{2 N}} \sqrt[4]{1+256\left(\frac{p}{p_{0}}\right)^{4 / 3}\left(\frac{\sigma^{2} X_{0}}{N \Delta x l^{2}}\right)^{2 / 3}},
$$

$\sigma$ is the single-measurement spatial RMS precision and $N$ the number of measurements. The high-momentum asymptote is found to have the same functional dependence on the detector parameters as was obtained with the back-of-the-envelope calculation of [2],

The method is also of interest for the high-precision measurement of muon momentum in large, finely-instrumented liquid argon detectors for long-range neutrino studies such as DUNE [15].

It is a pleasure to acknowledge the support of the French National Research Agency (ANR13-BS05-0002).

\section{References}

[1] HARPO, "hermetic argon polarimeter", http://llr.in2p3.fr/ dbernard/polar/harpo-t-p.html

[2] D. Bernard, "TPC in gamma-ray astronomy above pair-creation threshold," Nucl. Instrum. Meth. A 701, 225 (2013) Erratum: [Nucl. Instrum. Meth. A 713, 76 (2013)]. [arXiv:1211.1534 [astro-ph.IM]].

[3] D. Bernard, "Polarimetry of cosmic gamma-ray sources above $e^{+} e^{-}$pair creation threshold", Nucl. Instrum. Meth. A 729 (2013) 765. [arXiv:1307.3892 [astro-ph.IM]].

[4] P. Gros et al., "Measurement of polarisation asymmetry for gamma rays between 1.7 to $74 \mathrm{MeV}$ with the HARPO TPC", SPIE2016, 9905-95, arXiv:1606.09417 [astro-ph.IM].

[5] P. Gros et al., "Performance measurement of HARPO: a Time Projection Chamber as a gamma-ray telescope and polarimeter," arXiv:1706.06483 [astro-ph.IM], to appear in Astroparticle Physics.

[6] A. De Angelis et al. [e-ASTROGAM Collaboration], "The e-ASTROGAM mission,” Exper. Astron. 44 (2017) 25, [arXiv:1611.02232 [astro-ph.HE]].

[7] J. Perkins, "All-Sky Medium Energy Gamma-ray Observatory (AMEGO) - A discovery mission for the MeV gamma-ray band", presented at this Symposium.

[8] S. Takahashi et al., "GRAINE project: The first balloon-borne, emulsion gamma-ray telescope experiment," PTEP 2015, no. 4, $043 \mathrm{H} 01$ (2015).

[9] T. Tanimori et al., "Establishment of Imaging Spectroscopy of Nuclear Gamma-Rays based on Geometrical Optics," Sci. Rep. 7 (2017) 41511, [arXiv:1702.01483 [physics.ins-det]].

[10] G. Molière, "Theorie der Streuung schneller geladener Teilchen. III. Die Vielfachstreuung von Bahnspuren unter Berüksichtigung der statistischen Kopplung", Zeitschrift Naturforschung A 10 (1955) 177.

[11] C. Patrignani et al. [Particle Data Group], "Review of Particle Physics," Chin. Phys. C 40 (2016) 100001.

[12] R. Frühwirth, "Application of Kalman filtering to track and vertex fitting," Nucl. Instrum. Meth. A 262 (1987) 444. 
[13] M. Frosini and D. Bernard, "Charged particle tracking without magnetic field: optimal measurement of track momentum by a Bayesian analysis of the multiple measurements of deflections due to multiple scattering," Nucl. Instrum. Meth. A 867 (2017) 182, arXiv:1706.05863 [physics.data-an].

[14] P. Matisko and V. Havlena, "Noise covariance estimation for Kalman filter tuning using Bayesian approach and Monte Carlo”, Int. J. Adapt. Control Signal Process. 27 (2013) 957.

[15] R. Acciarri et al. [DUNE Collaboration], "Long-Baseline Neutrino Facility (LBNF) and Deep Underground Neutrino Experiment (DUNE) : Volume 4 The DUNE Detectors at LBNF," arXiv:1601.02984 [physics.ins-det]. 